\title{
Agencia de enfermeria en escolares con necesidades especiales
}

\section{Nursing agency for schoolchildren with special needs}

\author{
Gabriela Ramírez $^{1}$; ; Carlos Alberto Fernández-Silva ${ }^{2}$ (D)
}

Forma de citar: Ramírez G, Fernández-Silva CA. Agencia de enfermería en escolares con necesidades especiales. Salud UIS. 2021; e21033. doi: https://doi.org/10.18273/saluduis.53.e:21033 @ (c)

\section{Resumen}

Introducción: las instituciones escolares son espacios en los que se requiere la incursión del profesional de enfermería con el fin de fortalecer actividades de promoción de la salud y prevención de la enfermedad. A esto se suman actividades asistenciales de cuidado específico cuando se abordan escolares con necesidades especiales. Objetivo: analizar la necesidad de cuidados de enfermería en escolares con necesidades especiales en una institución educativa ubicada en el sur de Chile, según la teoría del déficit del autocuidado de Dorothea Orem y taxonomía diagnósticas de enfermería. Metodología: se tomó como base un registro anonimizado que contenía las características generales y de salud de 35 escolares con necesidades especiales, además del promedio de actividades de cuidado que se les realizó durante seis meses. Los datos se analizaron en forma cuantitativa descriptiva, y mediante su relación con la teoría del déficit del autocuidado de Dorothea Orem, y taxonomías diagnósticas de enfermería. Resultados: se evidenció en estudiantes diferentes condiciones complejas de salud que requerían en su mayoría sistemas parcial o totalmente compensatorios, al igual que diversas necesidades de cuidado no abordadas frente a problemas reales o de riesgo. Discusión: es evidente la necesidad de contar con profesionales de enfermería para abordar en forma integral tanto a estudiantes como a la comunidad educativa. Conclusiones: la presencia de profesionales de enfermería en establecimientos educativos permite brindar cuidados complejos, seguros y de calidad, que se logran gracias a la aplicación de sus fundamentos disciplinares.

Palabras clave: Servicios de Enfermería Escolar; Diagnósticos de Enfermería; Modelos de Enfermería; Enfermeras y Enfermeros; Rol de la Enfermera; Atención de Enfermería.

\begin{abstract}
Introduction: Educational institutions are spaces in which the incursion of the nursing professional is required to strengthen health promotion and disease prevention activities. Additionally, specific care assistance activities are included when schoolchildren with special needs are present. Objective: To analyze the need of nursing care in schoolchildren with special needs in an educational institution located in southern Chile, according to the selfcare deficit theory of Dorothea Orem and nursing diagnostic taxonomies. Methodology: An anonymized registry containing the general and health characteristics of 35 schoolchildren with special needs was taken as a basis, in addition to the average care activities that were carried out for 6 months. The data were analyzed in a descriptive quantitative way, and through its relation to Dorothea Orem's self-care deficit theory, and nursing diagnostic

1. Colegio de Enfermeras. Puerto Montt, Chile.

2. Universidad de Los Lagos. Puerto Montt, Chile.

Correspondencia: Carlos Alberto Fernández Silva. Dirección: Universidad de Los Lagos, Campus Puerto Montt - Chile. Chinquihue km 6, Móvil: +5 6956097752. Correo electrónico: carlosalberto.fernandez@ulagos.cl
\end{abstract}


taxonomies. Results: Different complex health conditions were evidenced in the students that mostly required partially or fully compensatory systems, as well as various unaddressed care needs facing real problems or risks. Discussion: The need for nursing professionals is evidenced to comprehensively address both students and the educational community. Conclusions: The presence of nursing professionals in educational establishments allows for the provision of complex, safe, and quality care, that are achieved thanks to the application of its disciplinary fundamentals.

Keywords: School Nursing; Nursing Diagnosis; Models; Nursing; Nurses; Nurse's Role; Nursing Care.

\section{Introducción}

La convención de los derechos del niño y de las personas con discapacidad hacen referencia a la necesidad de prevenir la violencia, y garantizar la salud, la educación, entre otros aspectos fundamentales para los seres humanos, se destaca que, en el caso de los y las menores con discapacidad, el contar con apoyo desde etapas tempranas de su vida favorece su desarrollo en las siguientes etapas de la vida ${ }^{1}$.

El contexto de la educación en poblaciones con necesidades especiales hace necesario recordar la definición del término discapacidad otorgado desde la Organización Mundial de la Salud(OMS): Discapacidad es un término general que abarca las deficiencias, las limitaciones de la actividad y las restricciones de la participación. Las deficiencias son problemas que afectan a una estructura o función corporal; las limitaciones de la actividad son dificultades para ejecutar acciones o tareas, y las restricciones de la participación son problemas para participar en situaciones vitales. Por consiguiente, la discapacidad es un fenómeno complejo que refleja una interacción entre las características del organismo humano y las características de la sociedad en la que vive ${ }^{2}$.

En Chile aproximadamente 7000 establecimientos educacionales desarrollan actividades de apoyo especializado a estudiantes con necesidades educativas especiales (NEE), sean estas transitorias o permanentes y estén o no asociadas a discapacidad, escenario que requiere del trabajo articulado entre el sector educación y salud ${ }^{3}$. Este tipo de educación se origina en el año 1982, cuando se crea una escuela pública para "sordomudos" en la Región Metropolitana, con el paso del tiempo se amplió la oferta de establecimientos y de cobertura, puesto que no sólo incluía a personas con discapacidad sensorial, sino también mental o motora ${ }^{4}$.

En sus inicios, este tipo de establecimientos eran exclusivos para menores en situación de discapacidad, ya que ésta era catalogada como algo patológico, en la actualidad se reconoce a esta condición como un aporte para la sociedad y los entornos educativos convencionales al favorecer la convivencia y la inclusión, visión que logró posicionarse gracias a los aportes de las ciencias sociales y de la pedagogía ${ }^{5}$.

Pese a los avances anteriormente mencionados, las necesidades sanitarias de los estudiantes de estos establecimientos no han sido cubiertas en su totalidad debido a que no se explicita en la legislación vigente ${ }^{6}$. Sin embargo, en el 2019 se publicó el decreto supremo $170^{7}$, en el cual se puede interpretar que las instituciones que atienden a este tipo de población tienen la facultad de solicitar los recursos materiales y humanos necesarios para garantizar la calidad de la educación, y así disminuir las barreras que se puedan presentar en el proceso ${ }^{8}$.

Lamentablemente para estas instituciones educativas, la relación con el ámbito de la salud mantiene un abordaje de tipo biomédico, desde el cual se atiende a las necesidades asistenciales evidentes, lo que conlleva a la vinculación de diferentes profesionales de la salud, entre quienes no se contempla al profesional de enfermería, se convierte así en un área emergente para la profesión.

La presencia de la/el enfermera/o escolar, y más aún en establecimientos en los que existen personas con necesidades educativas especiales, es de gran valor ya que su presencia es garantía de su abordaje integral, debido a que como gestores del cuidado: facilitan la continuidad de estos, establecen la necesidad de vincular a los profesionales, y el desarrollo de acciones de promoción y prevención en las que se vincula a toda la comunidad educativa ${ }^{9-12}$. Esto corresponde con los fundamentos epistemológicos de esta disciplina ${ }^{13}$.

El quehacer de los profesionales de enfermería garantiza el abordaje de al menos cuatro áreas tales como la asistencial, la educativa, la administrativa, y la de investigación. Con ello facilita dar respuesta a la satisfacción de necesidades, y garantiza la calidad 
de los cuidados ${ }^{10}$, lo que se potencia cuando en su quehacer cotidiano incorpora intencionadamente los paradigmas propios de su disciplina, y otros aspectos que contribuyen a su carácter científico como son el uso de taxonomías, entre ellas las propuestas por la NANDA (North American Nursing Diagnosis Association) ${ }^{14}$.

Por lo que para la presente investigación se tuvo presente la incorporación de las etiquetas diagnósticas NANDA, además del paradigma de enfermería propuesto por Dorothea Orem, denominado: Teoría del Déficit del autocuidado. En ella se plantean tres teorías: la teoría del autocuidado, la teoría del déficit del autocuidado y la teoría de los sistemas de enfermería, denominando al usuario como agente de autocuidado, y al profesional de enfermería como agencia de enfermería. Para Orem, los profesionales de enfermería aportan al bienestar de los individuos cuando se consigue el autocuidado, para ello, se vale de intervenciones de apoyo y educación, o de suplencia parcial o total en las actividades de autocuidado según sea el requerimiento de las personas ${ }^{15-16}$.

Es de mencionar que la institución educativa en la cual se llevó a cabo la investigación no cuenta con profesionales de enfermería, y las actividades de cuidado son aplicadas por cuidadores que no poseen la formación para realizar esta labor. Por tal razón se consideró pertinente trazar como objetivo general: analizar la necesidad de cuidados de enfermería en escolares con necesidades especiales en una institución educativa ubicada en el sur de Chile según la teoría del déficit del autocuidado y la taxonomía NANDA, y como objetivos específicos: describir a la población de estudiantes del centro educativo según características sociodemográficas y de salud; describir las actividades de cuidado que se realizan en forma cotidiana de acuerdo con la base de datos empleada en el centro educativo y por último, relacionar las actividades de cuidado con los requisitos del autocuidado de la Teoría del Déficit del autocuidado y con la taxonomía diagnósticas de la NANDA en su versión 2018-202014.

\section{Materiales y métodos}

Se realizó una investigación cuantitativa de tipo descriptiva en una institución educativa ubicada en el sur de Chile que tiene 120 estudiantes con necesidades educativas especiales, y quienes constituyen el universo, de ellos 35 generan la mayor demanda de cuidados según la información suministrada por el establecimiento.
Se contó con una base de datos que contiene el promedio mensual de las actividades de cuidado realizadas en los últimos seis meses (enero a agosto), a estudiantes catalogados como muestra para esta investigación; en forma complementaria se solicitó los datos relacionados con la salud y características sociodemográficas que permitiesen cumplir con los objetivos trazados. Esta información fue suministrada por la dirección del establecimiento en una hoja de Excel que no contenía los datos de identificación de los estudiantes, con el fin de resguardar su confidencialidad. Este proceso fue declarado en el protocolo de investigación presentado ante un comité ético científico, el cual dio su informe favorable.

Una vez recibida la información, se organizó en el mismo archivo para facilitar el proceso de análisis cuantitativo, posteriormente estos datos se analizaron con base en los conceptos de la teoría del déficit del autocuidado y de la taxonomía NANDA 2018-202014.

\section{Resultados}

De los 35 escolares, 21 fueron hombres y 14 mujeres, su promedio de edad fue de 14 años con una edad mínima 6 años y máxima de 23 , la mayoría se ubicó en el rango de los 6 a 15 años (54,3\%), seguido del rango de 16 a 20 años (34\%). Según su procedencia, 27 pertenecieron a la comuna donde está ubicada la escuela, 6 del límite urbano y 2 de otra comuna cercana.

En cuanto a su perfil de salud se pudo apreciar que el total de los estudiantes tiene alteraciones de su salud, en 15 de ellos se identificaron diferentes tipos de síndromes (Tabla1).

Tabla 1. Síndromes diagnosticados en la población de estudiantes

\begin{tabular}{lc}
\hline \multicolumn{1}{c}{ Síndrome } & Frecuencia \\
\hline Síndrome convulsivo & 3 \\
Síndrome de Cornelia de Lange & 2 \\
Síndrome de West & 2 \\
Síndrome de Down & 2 \\
Síndrome de Kabuki & 1 \\
Síndrome de Angelman & 1 \\
Síndorme Atáxico & 1 \\
Esquizencefalia de ángulo abierto & 1 \\
Artogriposis múltiple & 1 \\
Aganglionosis intestinal congénita & 1 \\
(enfermedad de Hirschprung) &
\end{tabular}


Las deficiencias intelectuales estuvieron presentes en este grupo de estudiantes, y predominan las clasificaciones moderada o severa en 33 de ellos (94\%). Se pudo establecer que la población evaluada presentó una o más alteraciones en los órganos de los sentidos, neuromotoras, del sistema nervioso central, neuropsiquiátricas, metabólicas y/o de la piel (Tabla 2).

Tabla 2. Alteraciones presentes en la población de estudiantes.

\begin{tabular}{lc}
\multicolumn{1}{c}{ Alteraciones } & Frecuencia \\
\hline En los órganos de los sentidos & 5 \\
Ceguera & 1 \\
Hipoacusia & \\
Neuro-motoras y otras del SNC & 4 \\
Epilepsia & 13 \\
Parálisis cerebral & 4 \\
Micro-hidrocelfalia & 8 \\
Plejías -paresias & 1 \\
Escoliosis & 1 \\
Mielomeningocele & \\
Neuropsiquiátricas & 2 \\
Autismo & 1 \\
Trastorno conductual & 1 \\
Psicosis & \\
Metabólicas & 1 \\
Resistencia a la insulina & 1 \\
Disfunción renal & 2 \\
Diabetes Mellitus tipo 1 & 1 \\
Hipertensión arterial & \\
De la piel & \\
Lesiones por presión grado 1 o 2 & 1 \\
Lesiones en área de pañal & \\
\hline &
\end{tabular}

El análisis de las actividades de cuidado diario arrojó como resultado la evidencia de diferentes niveles de complejidad, ya que estas incluyeron desde el cambio de pañales, hasta el cateterismo vesical o la administración de medicamentos (Tabla 3).

Tabla 3. Actividades de cuidado diario.

\begin{tabular}{lc}
\hline \multicolumn{1}{c}{ Actividad de cuidado } & $\begin{array}{c}\text { Promedio diario } \\
\text { Registro de 6 } \\
\text { meses }\end{array}$ \\
\hline $\begin{array}{l}\text { Aseo y confort (incluye cambio de } \\
\text { pañales 2 a 3 veces por día) }\end{array}$ & 58 \\
Alimentación asistida vía oral & 28 \\
Administración de medicamentos & 9 \\
Cateterismo vesical 2 a 3 veces por día & 3 \\
Apoyo para el uso del baño & 3 \\
Cambio de ropa (asistido o total) & 3 \\
Administración de insulina & 2 \\
\hline
\end{tabular}

Al relacionar las actividades de cuidado diario con la teoría del déficit del autocuidado de Dorothea Orem, se puede apreciar que éstas comprometen principalmente los requisitos de eliminación y excreción y de desviación de la salud, y hace necesario para la mayoría de ellos, la incorporación de un sistema totalmente compensatorio que permita la satisfacción de las necesidades de autocuidado cotidianas. (Tabla 4)

Tabla 4. Relación entre las actividades de cuidado diario y la teoría del Déficit del Autocuidado.

Tabla 4. Relación entre las actividades de cuidado diario y la teoría del Déficit del Autocuidado.

\section{Actividad de cuidado diario}

Aseo y confort

Alimentación asistida vía oral

Administración de medicamentos

Cateterismo vesical 2 a 3 veces por día

Apoyo para el uso del baño

Cambio de ropa (asistido o total)

Administración de insulina

\section{Requisito de autocuidado con el que se} relaciona

Requisito de autocuidado universal: Provisión de cuidados asociados con los procesos de eliminación y los excrementos.

Requisito de autocuidado universal: Mantenimiento de un aporte suficiente de Parcial y totalmente compensatorio. aire, agua y alimentos.

Requisito de autocuidado en desviación de la salud.

Requisito de autocuidado en desviación de la salud.

Requisito de autocuidado universal: Provisión de cuidados asociados con los procesos de eliminación y los excrementos.

Requisito de autocuidado universal: Provisión de cuidados asociados con los procesos de eliminación y los excrementos.

Requisito de autocuidado en desviación de la salud.
Sistema de enfermería empleado

Totalmente compensatorio

Totalmente compensatorio

Totalmente compensatorio

Parcialmente compensatorio

Parcialmente compensatorio

Totalmente compensatorio 
Al relacionar estas actividades con las etiquetas diagnósticas, se pudo establecer que estas aplican tanto para problemas reales como potenciales o de riesgo, pertenecientes principalmente a los dominios 1 (promoción de la salud), 3 (eliminación e intercambio) y 5 (autocuidado) (Tabla 5).

Tabla 5. Relación de las actividades de cuidado cotidiano con el lenguaje estandarizado

\begin{tabular}{|c|c|c|}
\hline Actividad de cuidado diario & Dominio & Etiquetas diagnósticas relacionadas \\
\hline \multirow{4}{*}{ Aseo y confort } & $\begin{array}{l}\text { Dominio 3: eliminación e } \\
\text { intercambio }\end{array}$ & $\begin{array}{l}00016 \text { Deterioro de la eliminación urinaria } \\
00023 \text { Retención urinaria }\end{array}$ \\
\hline & & 00196 Motilidad gastrointestinal disfuncional \\
\hline & & $\begin{array}{l}00197 \text { Riesgo de motilidad gastrointestinal } \\
\text { disfuncional }\end{array}$ \\
\hline & Dominio 5: Autocuidado & 00193: Descuido personal \\
\hline Alimentación asistida vía oral & Dominio 5: Autocuidado & (00102) Déficit de autocuidado: alimentación \\
\hline Administración de medicamentos & Dominio 1: promoción de la salud & $\begin{array}{l}\text { (00099) Mantenimiento ineficaz de la salud } \\
\text { (00078) Gestión ineficaz de la propia salud } \\
\text { (00043) Protección ineficaz }\end{array}$ \\
\hline Cateterismo vesical 2 a 3 veces & $\begin{array}{l}\text { Dominio 3: eliminación e } \\
\text { intercambio }\end{array}$ & $\begin{array}{l}\text { (00016) Deterioro de la eliminación urinaria } \\
\text { (00004) Riesgo de infección }\end{array}$ \\
\hline & Dominio 11: seguridad/protección & (00047) Riesgo de deterioro de la integridad cutánea \\
\hline Apoyo para el uso del baño & Dominio 4: actividad/ reposo & $\begin{array}{l}\text { Déficit de autocuidado: baño (00108) } \\
\text { Déficit de autocuidado: uso del inodoro (00109) }\end{array}$ \\
\hline Cambio de ropa (asistido o total) & Dominio 4: actividad/ reposo & Déficit de autocuidado: vestido (00109) \\
\hline
\end{tabular}

Como se mencionó en la metodología, las actividades analizadas correspondieron al registro de cuidados, no obstante, según la condición de salud de las estudiantes se consideró pertinente contemplar la posibilidad de uso de otras etiquetas diagnósticas pertenecientes a los dominios 1 (promoción de la salud) 5 (percepción/ cognición), 7 (rol/relaciones), 10 (principios vitales) y 11 (seguridad/protección) (Tabla 6).

Tabla 6. Etiquetas diagnósticas a considerar en el cuidado cotidiano

(00097) Disminución de la implicación en actividades recreativas (dominio 1, clase 1)

(00168) Sedentarismo (dominio 1, clase 1)

(00051) Deterioro de la comunicación verbal (dominio 5, clase 5)

(00061) Cansancio del rol de cuidador (dominio 7, clase 1)

(00062) Riesgo de cansancio de rol de cuidador (dominio 7, clase 1)

(00066) Sufrimiento espiritual (dominio 10, clase 3)

(00047) Riesgo de deterioro de la integridad cutánea (dominio 11, clase 2)

(00138) riesgo de violencia dirigida a otros(dominio 11, clase 3)

(00044) Deterioro de la integridad tisular (dominio 11,clase 2)

(00140) Riesgo de violencia autodirigida (dominio 11, clase 3)

(00039) Riesgo de aspiración (dominio 11, clase 2)

(00004) Riesgo de infección (dominio 11, clase 1)

(00155) Riesgo de caídas (dominio 11,clase 2)

(00035) riesgo de lesión (dominio 11, clase 2)

\section{Discusión}

Las características de salud de los estudiantes evaluados incluyen diversos síndromes y condiciones que ameritan la presencia de profesionales de enfermería, sin que este aspecto sea el único que lo justifique. Ya que sus acciones desde la perspectiva asistencial en un entorno educativo van más allá de garantizar la cobertura de necesidades que se evidencian en esta población: se posibilita el abordaje de toda la comunidad educativa (docentes, personal administrativo, padres, madres, apoderados), mediante acciones de promoción y prevención, de esta forma se favorece la detección temprana de afecciones de la salud, y la protección específica frente a enfermedades que se pueden prevenir por medio de actividades como, por ejemplo, la vacunación ${ }^{17-20}$.

Desde las acciones administrativas y de gestión, los profesionales de enfermería también contribuyen con la construcción de procesos que buscan garantizar la seguridad y continuidad en la atención como parte de la calidad de sus cuidados ${ }^{21,22}$. Esto conlleva, entre otras acciones, a establecer formas de comunicación oficial con instituciones de la red asistencial en caso 
de ser requerido ante complicaciones de la salud de los escolares, es factible que esto ocurra, si se tiene en cuenta su perfil de salud.

En consideración del modelo teórico empleado, se pudo establecer la necesidad de sistemas parcial o totalmente compensatorios para el desarrollo de actividades de autocuidado; esto requiere del criterio del profesional de enfermería para discernir cuáles de ellas pueden ser delegadas o ejecutadas bajo su responsabilidad, ya que este es un aspecto que hace parte de la gestión del cuidado ${ }^{23,24}$.

En relación con el análisis mediante dominios y etiquetas diagnósticas de enfermería, cabe mencionar que incorporarlo en la planificación de cuidados facilita la visualización de necesidades de cuidado que se suelen pasar por alto en una práctica de cuidado instintiva. Esto es un aporte fundamental de la práctica profesional de enfermería al posibilitar la valoración integral de las necesidades que involucran esferas como la psicosocial y espiritual, además es consecuente con el Modelo de Atención Integral de Salud Familiar con enfoque biopsicosocial, declarado como paradigma en el contexto sanitario chileno ${ }^{25,26}$.

El uso del lenguaje estandarizado permite además generar la necesidad de intervenciones que no sólo involucran a los profesionales sanitarios, sino también a educadores físicos, que podrían aportar al desarrollo de actividades que se adecúen en forma pertinente a la condición de salud de los estudiantes, sin embargo, a nivel país, la aplicación de este tipo de lenguaje disciplinar es escaso ${ }^{27}$.

\section{Conclusiones}

Debido a las características de los estudiantes se requiere realizar actividades de cuidado dependiente, escenario en el cual la presencia constante de la agencia de enfermería es requerida con el objetivo de resguardar la calidad de los cuidados y la gestión del cuidado. A partir de esto se genera la necesidad de establecer el diagnóstico integral de necesidades y establecer las medidas de cuidado pertinentes en las cuales se vincula a otros profesionales, con el fin de lograr el bienestar de los usuarios.

La presencia de un profesional de enfermería garantizaría el abordaje del entorno, incorporando desde esta perspectiva a todas las personas que integran la comunidad educativa y los establecimientos de la red, en pro de la garantía en la continuidad de los cuidados.
Esto también otorgaría la complejidad necesaria para contribuir a la seguridad y calidad de los mismos, y por ende al bienestar de los sujetos que los reciben, más aún, si se tiene en cuenta el complejo escenario epidemiológico generado raíz virus SARS-CoV-2 (COVID 19).

Se requiere desarrollar investigaciones que involucren además de la población de escolares, a los demás miembros de la comunidad educativa, tal como educadores, cuidadores, personal administrativo, y familias, para, de esta manera poder contar con mayor evidencia que facilite la implementación de medidas tendientes al bienestar colectivo.

\section{Limitaciones}

Cabe mencionar que si bien los resultados de la presente investigación permiten reflejar una realidad que puede presentarse en otros establecimientos educativos en los que se atienden escolares con características especiales, se requeriría de otros diseños metodológicos para poder constituir evidencia que permita inferir los resultados. De igual manera, se reconoce como limitante dentro del análisis realizado, la omisión de la taxonomía NIC.

\section{Consideraciones éticas}

El presente trabajo contó con la autorización de la dirección del establecimiento, y con el informe favorable de Comité Ético Científico del Servicio de Salud del Reloncaví, ubicado en la ciudad de Puerto Montt, Chile (ordinario 103-2020).

\section{Conflictos de interés}

No se declara conflictos de interés.

\section{Referencias}

1. Organización Mundial de la Salud - Unicef. El desarrollo del niño en la primera infancia y la discapacidad. Un documento de debate. Malta: OPS; 2013. $41 \mathrm{p}$.

2. Organización Mundial de la Salud. Temas de salud: Discapacidades. https://www.who.int/topics/ disabilities/es/

3. Ministerio de Educación de Chile. Educación especial. http://escolar.mineduc.cl/educacionespecial/ 
4. Bravo P. Ley 20.422 sobre Discapacidad en Chile: análisis y aplicación a comunidades Sordas https:// www.academia.edu/22359312/Ley_20.422_sobre_ Discapacidad_en_Chile_an\%C3\%A1lisis_y_ aplicaci\%C3\%B3n_a_comunidades_Sordas

5. Ministerio de Educación de Chile. Guías: escuela, familia y discapacidad. https://especial.mineduc.cl/ recursos-apoyo-al-aprendizaje/recursos-la-familia/ guias-escuela-familia-discapacidad/

6. Normas sobre igualdad de oportunidades e inclusión social de personas con discapacidad/Ley 20.422 . Santiago: Ministerio de Planificación de Chile; 10 de febrero de 2010, modificada: 7 jul 2020 decreto 298.

7. Normas para determinar los alumnos con necesidades educativas especiales que sarán beneficiarios de las subvenciones para educación especial/Decreto 170. Santiago: Ministerio de Educación Chile; 21 de abril 2010, última versión: 25 de agosto de 2010.

8. Shani C, Ortega J, Rodríguez MJ. Políticas Educativas y Educación especial en Chile (1990 a 2010). [Tesis]. Universidad academia de humanismo cristiano - Facultad de pedagogía: Santiago; 2013.

9. Navarro M. Enfermería escolar: una revisión bibliográfica [Trabajo de grado]. Universitat Rovira I Virgili: España; 2016.

10. Ortiz MM. Rol de la enfermera escolar en los colegios de educación especial. Rev Fund Salud Infantil. 2011; 2: 48-50.

11. Colegio oficial de enfermería en Madrid. Enfermería escolar, la gran desconocida. [Actualizado 31 de octubre 2013] https://www.codem.es/actualidad/ enfermeria-escolar-gran-desconocida

12. SOCHIESE. ¿Quiénes son las enfermeras escolares?. Disponible en: http://www.sochiese.cl/

13. Amezcua, Ml. ¿Por qué afirmamos que la Enfermería es una disciplina consolidada? Index Enferm. 2018; 27(4): 188-190.

14. Hederman H, Kamitsuru S [Ed]. Diagnósticos enfermeros. Definiciones y clasificación 2018-2020. Barcelona: Elsevier. 2019, 700p.

15. Naranjo Y, Concepción JA, Rodríguez M. La teoría del déficit de autocuidado: Dorothea Elizabeth Orem. GME 2017; 19(3).

16. Prado LA, González M, Paz N, Romero K. La teoría Déficit de autocuidado: Dorothea Orem punto de partida para calidad en la atención. Rev Med Electron. 2014; 36(6): 835-845.

17. Lagoueyte Gómez MI. El cuidado de enfermería a los grupos humanos. Rev Univ Ind Santander Salud 2015; 47 (2): 209-2013.
18. Segura I, Barrera L. Un llamado a Enfermería para responder al cuidado de la salud de las personas en situación de enfermedad crónica por su impacto en su calidad de vida. Salud Uninorte 2016; 32(2): 228-243.

19. Castañeda C, Romero K. La Atención Primaria de Salud y la Enfermería. Episteme 2016; 3(3): 384-401.

20. Bernárdez S. El papel del personal de enfermería en la promoción e implementación de las vacunaciones. Vacunas. 2018; 19 (1): 31-36.

21. Febré N, Mondaca-Gómez K, Méndez-Celis $P$, Badilla-Morales V, Soto-Parada P, Ivanovic P, et al. Calidad en enfermería: su gestión, implementación y medición. Rev Med Clin Condes. 2018; 29(3): 278-287. doi: https://doi.org/10.1016/j. rmclc.2018.04.008

22. Alonso da Costa BNA, de Andrade SR, Soares CF, Ballesteros Pérez EI, Capilla S, Bernardino E. The continuity of hospital nursing care for Primary Health Care in Spain. Rev Esc Enferm USP. 2019; 53: e03477. doi: http://dx.doi.org/10.1590/S1980220X2018017803477

23. Milos P, Bórquez PB, Larraín AI. La "Gestión del Cuidado" en la legislación chilena: interpretación y alcance. Cienc. Enferm. 2010; 16 (1):17-29.

24. Ferreira Gímerson E, Dall'Agnol CM, Porto Adrize R. Repercussões da proatividade no gerenciamento do cuidado: Percepções de enfermeiros. Esc Anna Nery. 2016; 20 (3): e20160057. doi: http://dx.doi. org/10.5935/1414-8145.20160057

25. Hernández R, Moreno MG, Cheverría S, Landeros M, Interial MG. Cuidado de enfermería perdido en pacientes hospitalizados en una institución pública y una privada. Index Enferm. 2017; 26 (3): 142-146.

26. Parra D. Revolución científica de la salud familiar en Chile. Revenf 2016; 31: 243-253. Doi: http:// dx.doi.org/10.15517/revenf.v0i31.23159

27. Campos C, Jaimovich S, Wigodski J, Aedo V. Conocimientos y uso clínico de la metodología enfermera (NANDA, NIC, NOC) en enfermeras/ os que trabajan en Chile. Rev Iberoam Educ Investi Enferm. 2017; 7(1): 33-42. 